\title{
The role of diagnostic hysteroscopy in diagnosis of incomplete uterine septum in patients with recurrent pregnancy loss in the era of transvaginal 3D ultrasound scan
}

\author{
O. Abuzeid ${ }^{1 *}$ (D) J. LaChance ${ }^{2}$, J. Hebert ${ }^{1}$, M. I. Abuzeid ${ }^{1,3,4}$ and R. Welch ${ }^{1,5}$
}

\begin{abstract}
Purpose: To determine the accuracy of transvaginal 3D ultrasound scan (TV 3D US) in detecting partial septate uterus (PSU) in patients with recurrent pregnancy loss (RPL).

Methods: This retrospective study included 113 patients with an initial diagnosis of unexplained RPL, who were subsequently found to have PSU on diagnostic hysteroscopy and who had TV 3D US prior to surgery. The diagnosis of PSU was made at the time of a diagnostic hysteroscopy based on ESHRE-ESGE classification of Müllerian anomalies. Based on hysteroscopic findings, patients were divided into two groups: those with PSU and a central point of indentation at an acute angle $<90^{\circ}$ (PSUAA) [30.1\%], and those who had PSU and a central point of indentation at an obtuse angle (PSUOA) [69.9\%]. We compared the mean internal indentation length at the fundal midline (IILFM) in millimeters on TV 3D US and on diagnostic hysteroscopy. For the purpose of this study, a diagnosis of PSU on hysteroscopy was made if IILFM measured $\geq 10 \mathrm{~mm}$.

Results: The mean IILFM (mm) on hysteroscopy was significantly higher than the mean IILFM (mm) measured on TV 3D US in patients with PSUAA (18.5 \pm 6.5 vs $4.9 \pm 4.4$; $P<0.001)$, in patients with PSUOA (14.1 \pm 3.8 vs $4.3 \pm 3.4$; $P<0.001)$, and in the overall population $(15.3 \pm 5.1$ vs $4.1 \pm 4.4 ; P<0.001)$.

Conclusions: The data suggest that mean IILFM in patients with RPL and PSU can be underestimated on TV 3D US. Therefore, its diagnostic accuracy in such patients may need further evaluation.
\end{abstract}

Keywords: Hysteroscopy, Recurrent pregnancy loss, Partial septate uterus, TV 3D US

\section{Introduction}

Unexplained factors account for more than $50 \%$ of the etiology of RPL $[1,2]$. A large number of these patients are advised to keep trying, have IVF with pre-implantation genetic screening (PGS), or are referred for evaluation for immunological factors of RPL [3]. Such treatment options are expensive and may not be successful, especially if the actual underlying etiology is missed [3]. In addition, in patients with idiopathic RPL, the work by Murugappan et al. [4] suggested that expectant management of RPL is as

\footnotetext{
* Correspondence: omabuzeid@gmail.com

${ }^{1}$ Department of Obstetrics and Gynecology, Hurley Medical Center and Michigan State University College of Human Medicine, Flint Campus, Flint, Michigan, USA

Full list of author information is available at the end of the article
}

successful as IVF-ET with PGS and had a lower median time to pregnancy [4]. However, one recent study reported a $41.1 \%$ incidence of abnormal embryonic karyotype in patients who had a negative work-up for RPL, suggesting the true incidence of unexplained RPL to be approximately $24.5 \%$ [5]. In addition, a recent study by HodesWertz et al. [6] suggested that unexplained RPL is mostly caused by chromosomal abnormalities, with only a residual $6.9 \%$ miscarriage rate [6].

The prevalence of uterine anomalies in patients with RPL when optimal tests, such as TV 3D US, are used has been estimated to be $13.3 \%$ [7]. Septate uterus is the most common anomaly in this population accounting for approximately $50 \%$ [7, 8]. Sugiura-Ogasawara et al. [5] suggested that major uterine anomalies have a negative impact on the 
reproductive outcome in patients with RPL [5]. Once such anomalies are discovered and surgically corrected, $70-80 \%$ of patients achieve a successful live birth [9].

Current literature suggests high sensitivity and specificity of TV 3D US in the diagnosis of uterine septum in patients with RPL $[10,11]$. On the other hand, in many parts of the world, especially in developing countries, TV 3D US is not commonly used during the work-up of patients with RPL. Instead, other radiological tests such as TV 2D US and hysterosalpingogram (HSG) are being used in screening for such anomalies. However, HSG can only detect significant fundal indentation, but it cannot differentiate between septate and bicornuate uterus. Similarly, TV 2D US can detect a significant endometrial separation in the transverse plane, but cannot differentiate between septate and bicornuate uterus. In addition, limited data published by our group suggest that HSG and TV 2D US are not accurate in detecting subtle fundal indentation in patients with RPL or infertility [12, 13]. Furthermore, some work by our group showed that even TV 3D US may not be accurate in detecting such subtle fundal indentation in patients with RPL or infertility [13-16]. If a diagnosis of such uterine anomalies is missed by the abovementioned radiological tests, such patients may be categorized under unexplained RPL. Therefore, some investigators suggest that a diagnostic hysteroscopy should be part of the work-up of RPL, especially to rule out subtle uterine anomalies such as PSU [12-16]. The aim of this study is to compare the accuracy of detecting PSU in patients with unexplained RPL when using TV 3D US versus hysteroscopy. This was determined by comparing the internal indentation length at the fundal midline (IILFM) on TV 3D US to the actual length measured during a diagnostic hysteroscopy.

\section{Materials and methods}

This is a retrospective study of all patients who were found to have PSU on a diagnostic hysteroscopy performed, as part of their work-up for unexplained RPL, at our unit between January 1, 2008, and October 31, 2017. RPL was defined as two or more consecutive miscarriages. All patients in this study underwent a complete work-up for RPL. This work-up included extensive history, a thorough physical examination, and investigations to determine any structural genetic, endocrinologic, anatomic, immunologic, microbiologic, and inherited thrombophilic factors. We inquired about iatrogenic factors including a review of tobacco, alcohol, and caffeine use and a review of exposure to toxins and chemicals. Uterine factors were evaluated with transvaginal 3D US with and without saline sonohysterogram and diagnostic hysteroscopy. A limited immunologic work-up including lupus anticoagulant antibodies, anticardiolipin antibodies (IgG/IgM), and B2-glycoprotein-1 antibodies (IgG/IgM) was done. A thrombophilia profile including factor V Leiden, prothrombin gene, fasting homocysteine, antithrombin activity, protein $\mathrm{C}$ activity, and protein $\mathrm{S}$ activity was ordered. Work-up for endocrine factors included thyroid-stimulating hormone, prolactin, and fasting insulin and glucose levels. Serum follicle-stimulating hormone, luteinizing hormone was ordered. Tests for parental structural chromosome rearrangement and cervical/vaginal cultures were done. The majority of these patients also complained of secondary infertility. The study received an exemption from the Institutional Review Board at Hurley Medical Center, Flint, Michigan. The inclusion criterion was to have undergone a TV 3D US prior to a diagnostic hysteroscopy, both done at our unit. Patients who had associated uterine fibroids, endometrial polyps, or intrauterine scar tissue and those with complete septum were excluded. Also, all patients who were found to have an underlying etiology for their RPL were excluded from this study. The study included 113 patients who fulfilled such inclusion criteria whose work-up suggested unexplained RPL and who were found to have PSU on hysteroscopy.

A diagnostic hysteroscopy was performed under modified general anesthesia at the mid-follicular phase of the menstrual cycle or while the patient was on oral contraceptives. Medications commonly used included Propofol, Versed, and Fentanyl. Hysteroscopy combined with a laparoscopic procedure were performed under general endotracheal anesthesia. All hysteroscopies were performed by one surgeon (MIA). An ACMI hysteroscope (Division of Olympus; Maple Grove, MN, USA) was used in all patients. Normal saline was used as a distension medium during the initial diagnostic hysteroscopy. Once the diagnosis of PSU was made, Glycine $1.5 \%$ was used and hysteroscopic division of the septum was performed at the same setting as described before [17]. At the time of diagnostic hysteroscopy, the type of uterine anomaly was documented and described according to ASRM classification [18]. The population was classified based on hysteroscopic findings into patients with incomplete uterine septum (34 patients, 30.1\%) [Class Vb] and those with significant arcuate uterine anomaly (79 patients, 69.9\%) [Class VI]. For the purpose of this study, a diagnosis of an incomplete uterine septum was made, if the central point of indentation was at an acute angle $(<$ $90^{\circ}$ ), did not reach to the region of the internal or external cervical os, and the IILFM measured $\geq 10 \mathrm{~mm}$, while if the central point of indentation was at obtuse angle $\left(>90^{\circ}\right)$ and the IILFM was $\geq 10 \mathrm{~mm}$, the diagnosis of a significant arcuate uterine anomaly was made [19]. In 2013, a new classification of Müllerian anomalies was proposed by the European Society of Human Reproduction and Embryology and the European Society for Gynecological Endoscopy (ESHRE-ESGE) [20]. In this classification, the definition of PSU is made if there is an IILFM $>50 \%$ of myometrial wall thickness in the fundal region irrespective 
of the appearance of the central point of indentation [20]. It is now acknowledged by many practitioners that the ESHRE-ESGE classification is more useful in clinical practice than the American Society of Reproductive Medicine (ASRM) classification. Therefore, we re-classified the population in our study according to the ESHRE-ESGE classification. In doing so, all patients with incomplete uterine septum or significant arcuate uterine anomaly were re-named PSU. The population was classified based on hysteroscopic findings into patients who have PSU with the central point of indentation at an acute angle $<90^{\circ}$ (PSUAA) [34 patients, 30.1\%] and those who have PSU with the central point of indentation at an obtuse angle (PSUOA) [79 patients, 69.9\%].

All patients in our study underwent a diagnostic hysteroscopy as part of the work-up for RPL. If PSU was suspected, the actual internal indentation length at fundal midline (IILFM) was measured indirectly at the time of diagnostic hysteroscopy by advancing a straight loop electrode on one side of the internal indentation up to the tubal ostium. The IILFM was then indirectly calculated to be approximately $60 \%$ of that length (according to Pythagorean Theorem in Mathematics). The actual internal indentation length at fundal midline was measured directly after the septum was divided by comparing its length to the length of the black cutting metal wire $(5 \mathrm{~mm})$ and yellow insulator portion $(10 \mathrm{~mm})$ of the straight resectoscope loop electrode of the ACMI hysteroscope (Fig. 1, Fig. 2) [17]. In our study, we used the data of the direct measurement of IILFM as all patients underwent septum division at the same setting.

Before hysteroscopy, an initial TV 2D US assessment of the pelvis was performed, as part of RPL work-up, to determine the presence of any adnexal pathology such as endometrioma, hydrosalpinx, or any uterine pathology, such as uterine fibroid and endometrial polyps. This was followed by a TV 3D US utilizing a Medison Sonace 8000 US machine (Medison, Seoul 135-280, Korea) and an endocavitary probe RIC5-9H 5-9 MHz. The uterus was then visualized by TV 3D US in a strict mid-sagittal view, adjusting the capture window to obtain the optimal 3D US volume. The 3D US volume was then obtained, using the automatic sweep of the mechanical transducer, at angle of $90^{\circ}$ from one side of the uterus to the other, bisecting the capture plane. If there was any doubt about the quality or completeness of the TV 3D US volume, the acquisition was repeated. The dataset was then stored to the hard drive of the ultrasound machine. The dataset was adjusted to ensure that the endometrium was evident in the three planes, the fundal aspect of the uterus and the endometrial cavity were demonstrable in the coronal plane, and the interstitial portions of both fallopian tubes were displayed simultaneously. This enabled us to assess the uterine morphology and shape of the uterine cavity in the coronal plane. Any internal indentation in the endometrial cavity was calculated on TV 3D US. This involved measuring the distance from the midpoint of the line joining the internal tubal ostia and the bottom of indentation of the cavity (Fig. 3a and Fig. 4a with their corresponding hysteroscopic appearance, Fig. 3b and Fig. 4b). Based on IILFM on TV 3D US, the patients were divided into four subgroups. The IILFM of subgroups $1,2,3$, and 4 were $0.00 \mathrm{~mm}, 1-4.9 \mathrm{~mm}, 5-9.9 \mathrm{~mm}$, and $\geq 10 \mathrm{~mm}$ respectively. We compared the IILFM on hysteroscopy to the IILFM on TV 3D US in patients with PSUAA, with PSUOA, and in the overall population. We also calculated the incidence of all subgroups (1-4) in patients with PSUAA, with PSUOA, and in the overall population. Statistical analysis was performed using Student's paired $t$ test, chi-square analysis, and correlation analysis where appropriate.

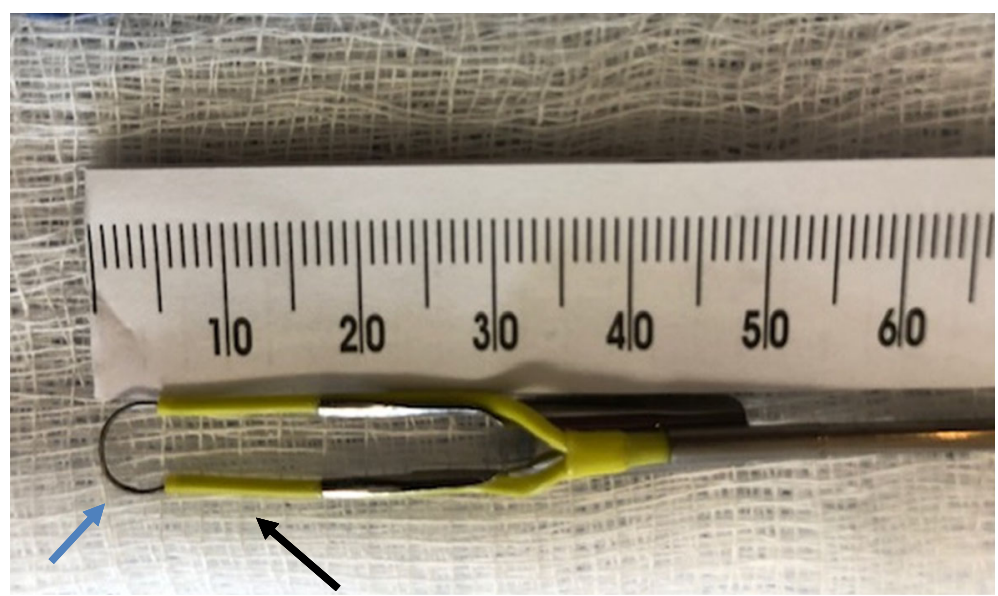

Fig. 1 The length of various parts of the straight resectoscope loop electrode of the ACMI hysteroscope that was used to measure IILFM. The black cutting metal wire of the straight resectoscope loop electrode is $5 \mathrm{~mm}$ in length (blue arrow), while the yellow insulating portion of the resectoscope loop is $10 \mathrm{~mm}$ in length (black arrow) 


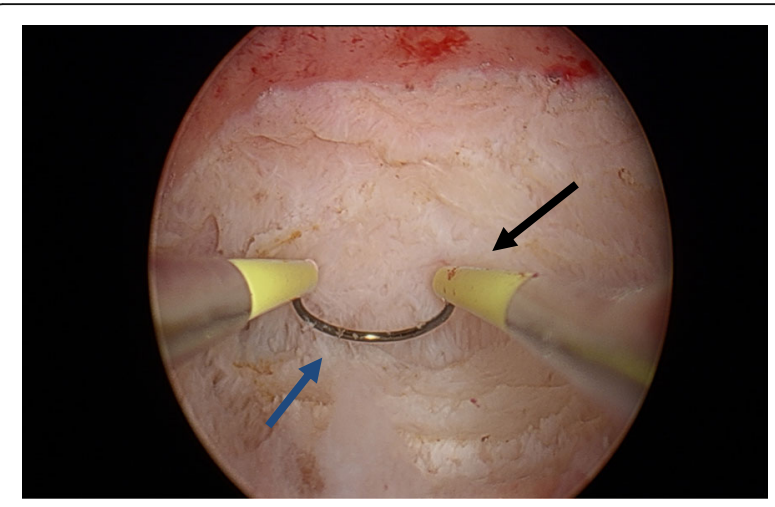

Fig. 2 How a straight resectoscope loop electrode was used to directly measure the IILFM (blue arrow is pointed to the metal electrode, $5 \mathrm{~mm}$ in length) (black arrow is pointed to the yellow insulator, $10 \mathrm{~mm}$ in length)
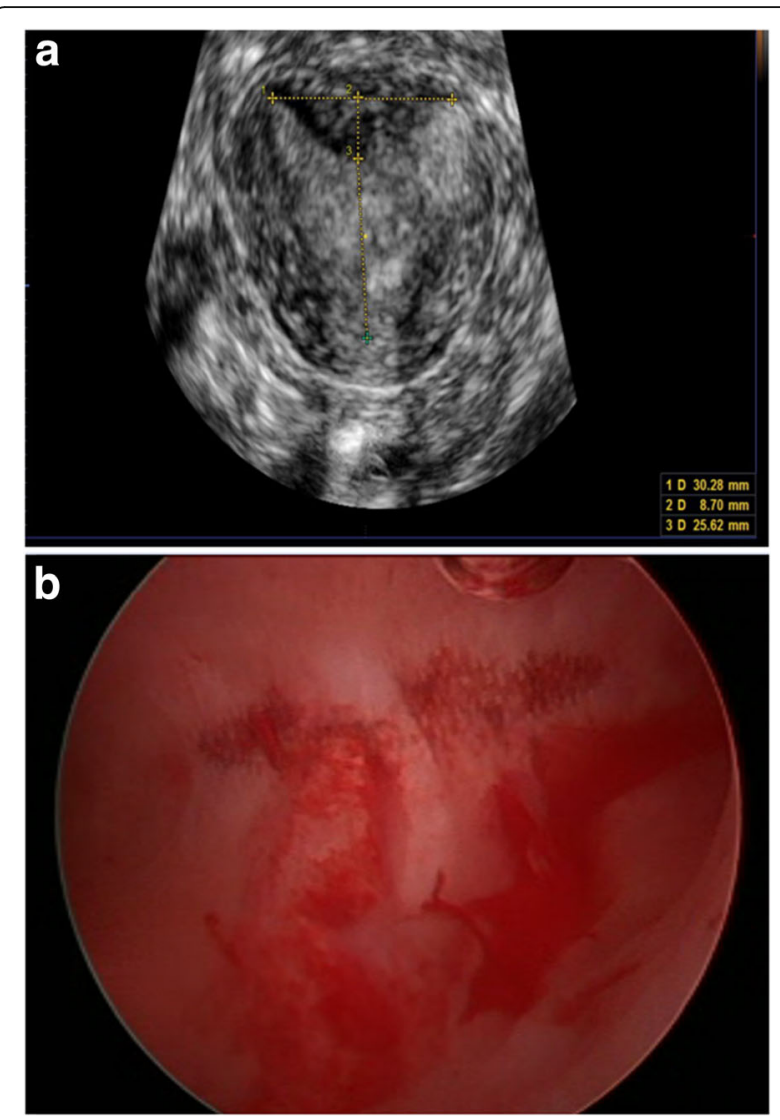

Fig. 3 a How the IILFM was calculated on TV 3D US by measuring the distance from the midpoint of the line joining the internal tubal ostia and the central point of indentation in a patient with a PSUAA. b The corresponding hysteroscopic appearance of the uterine fundus of the patient with PSUAA whose TV 3D US is illustrated in a

\section{Results}

Table 1 summarizes background details (Table 1). The majority of patients $(93.8 \%)$ presented with secondary infertility in addition to RPL. The mean IILFM measured in millimeters on hysteroscopy was significantly higher than the IILFM measured on TV 3D US, in patients with PSUAA $(18.5 \pm 6.5$ vs $6.3 \pm 6.3 ; P<0.001)$, in patients with PSUOA (14.1 \pm 3.8 vs $4.3 \pm 3.4 ; P<0.001)$, and in the overall population $(15.3 \pm 5.1$ vs $4.9 \pm 4.4 ; P<0.001)$ [Fig. 5]. A correlation analysis between the findings on hysteroscopy and the findings on TV 3D US revealed a moderate correlation for PSUAA $(r=0.596, P=0.001)$, a weak correlation for patients with PSUOA $(r=0.279$, $P=0.016)$, and a moderate correlation for the overall population $(r=0.481, P<0.001)$.

Table 2 illustrates the incidence of four subgroups (14) based on the IILFM on TV 3D US in patients with PSUAA, with PSUOA, and in the overall population with IILFM $\geq 10 \mathrm{~mm}$ on hysteroscopy (Table 2). There was no evidence of any IILFM $(0.00 \mathrm{~mm})$ on TV 3D US

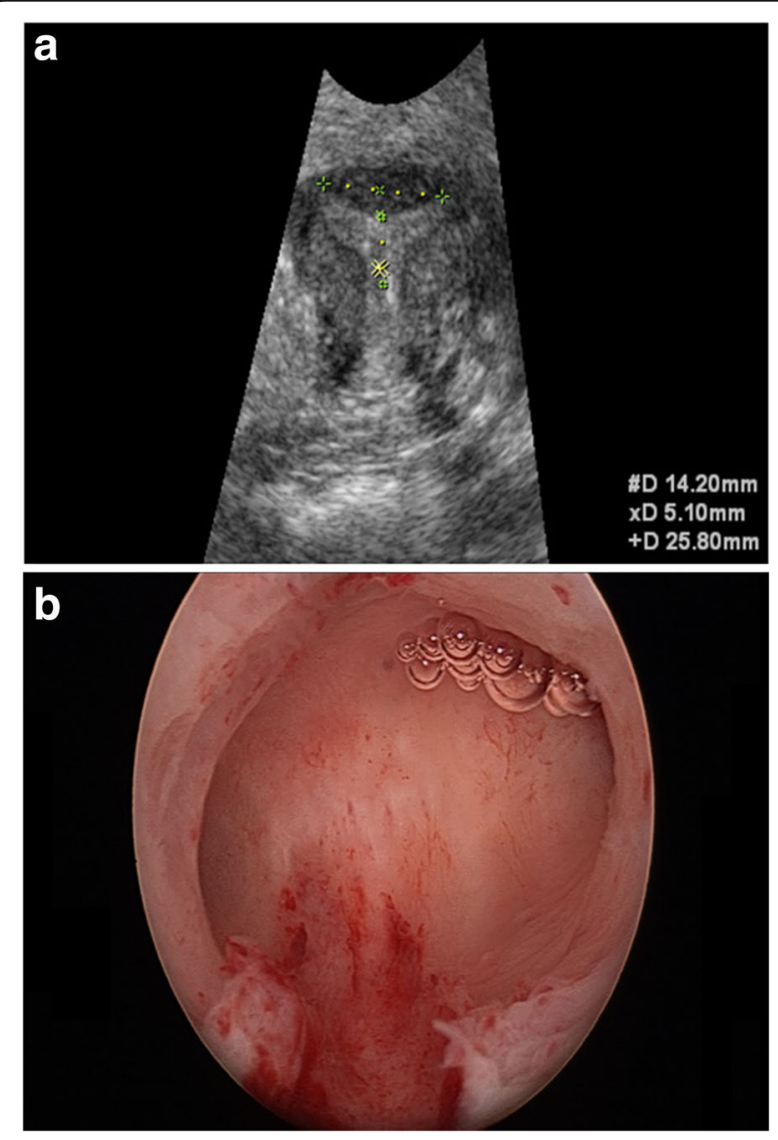

Fig. 4 a How the IILFM was calculated on TV 3D US by measuring the distance from the midpoint of the line joining the internal tubal ostia and the central point of indentation in a patient with a PSUOA. b The corresponding hysteroscopic appearance of the uterine fundus of the patient with PSUOA whose TV 3D US is illustrated in a 
Table 1 Background details

\begin{tabular}{|c|c|c|c|c|}
\hline & PSUAA, no. $=34$ & PSUOA, no. $=79$ & $P$ value & Overall total population, no. $=113$ \\
\hline Age (years) & $32.2 \pm 5.8$ & $33.9 \pm 5.7$ & NS & $33.4 \pm 5.7$ \\
\hline Duration of Infertility (years) & $2.3 \pm 1.3$ & $2.8 \pm 2.3$ & NS & $2.6 \pm 2.1$ \\
\hline$\%$ secondary infertility & $94.1 \%$ & $93.7 \%$ & NS & $93.8 \%$ \\
\hline $\mathrm{BMI}\left(\mathrm{kg} / \mathrm{m}^{2}\right)$ & $27.2 \pm 5.3$ & $28.2 \pm 6.8$ & NS & $27.9 \pm 6.4$ \\
\hline Day 3 FSH (mlU/mL) & $6.8 \pm 1.7$ & $7.3 \pm 3.1$ & NS & $7.2 \pm 2.8$ \\
\hline Day $3 \mathrm{LH}(\mathrm{mlU} / \mathrm{mL})$ & $5.7 \pm 3.5$ & $5.7 \pm 3.6$ & NS & $5.7 \pm 3.6$ \\
\hline Prolactin $(\mathrm{ng} / \mathrm{mL})$ & $10.5 \pm 4.3$ & $13.2 \pm 8.8$ & NS & $12.4 \pm 7.6$ \\
\hline TSH (mIU/L) & $1.9 \pm 1.3$ & $1.6 \pm 0.8$ & NS & $1.7 \pm 1.0$ \\
\hline No. of miscarriages & $2.6 \pm 0.9$ & $3.1 \pm 1.4$ & NS & $2.9 \pm 1.3$ \\
\hline$\%$ male infertility & $20.6 \%$ & $10.1 \%$ & NS & $13.3 \%$ \\
\hline$\%$ ovulatory disorder & $20.6 \%$ & $12.7 \%$ & NS & $15.0 \%$ \\
\hline$\%$ tubal factors & $15.6 \%$ & $22.5 \%$ & NS & $20.4 \%$ \\
\hline$\%$ endometriosis & $21.8 \%$ & $25.7 \%$ & NS & $26.5 \%$ \\
\hline
\end{tabular}

in 31 patients (27.4\%) [subgroup 1]. The IILFM on TV 3D US was found to be $1-4.9 \mathrm{~mm}$ in 19 patients $(16.8 \%)$ [subgroup 2]. The IILFM on TV 3D US was found to be $5-9.9 \mathrm{~mm}$ in 42 patients (37.2\%) [subgroup 3]. The internal IILFM on TV 3D US was found to be $\geq 10 \mathrm{~mm}$ in 21 patients $(18.6 \%)$ [subgroup 4 ]. There is a statistically significant difference in the proportion of subgroups 1-4 in patients with PSUAA and with PSUOA $(P<0.01)$. There is a statistically significant difference in proportion of PSUAA (20.6\%) and PSUOA (44.3\%) in subgroup 3 (IILFM between 5 and $9.9 \mathrm{~mm})[P<0.01]$. Also, there is a statistically significant difference in proportion of PSUAA (35.8\%) and PSUOA (11.4\%) in subgroup 4 (10 or greater) $[P<0.01]$. Most PSUOA found $(88.6 \%)$ were less than $10 \mathrm{~mm}$ compared to $64.7 \%$ of PSUAA found (Table 2).

\section{Discussion}

Uterine septum accounts for approximately 13.3\% of causes of RPL [7]. Hysteroscopic surgical correction of such anomalies has proven to be very successful in achieving live birth in these patients, albeit there is no prospective randomized controlled study to prove this notion until now [9]. The data in this study suggest that in many patients with RPL, who were thought to have unexplained factors, such anomalies were not identified by TV 3D US. The latter has been used as the gold standard for diagnosis of such anomalies [10, 11]. A negative TV 3D US in patients with RPL, in the absence of any other cause of RPL, places these patients in the category of unexplained RPL. Many of such patients are offered IVF-ET with PGS, referred to a reproductive immunology unit, or are encouraged to keep trying to conceive on their own [3]. On the other hand, our data suggest that a diagnostic hysteroscopy by an experienced reproductive surgeon may help in detecting PSU in some patients with RPL with less conclusive findings on TV 3D US. Therefore, we suggest that it should be a part of routine work-up for RPL after a TV 3D US is done, even if the latter was negative for PSU.

Diagnostic hysteroscopy alone, without laparoscopy for evaluation of the external contour of the uterus, cannot differentiate between septate and bicornuate uterus and should only be performed after TV 3D US is done in such patients, especially if one is planning to proceed with operative hysteroscopy at the same setting. In addition, it should be emphasized that TV 3D US is an essential part of work-up of RPL. Its role is imperative, especially when combined with $\mathrm{SIH}$, in the diagnosis of submucosal fibroid, endometrial polyps and partial

Table 2 Incidence of four subgroups (1-4) based on the IILFM on TV 3D US in patients with PSUAA, with PSUOA, and in the overall population with IILFM $\geq 10 \mathrm{~mm}$ on hysteroscopy

\begin{tabular}{llll}
\hline & PSUAA, no. $=34$ & PSUOA, no. $=79$ & Overall total population, no. = 113 \\
\hline Subgroup 1, ILLFM $(0.00 \mathrm{~mm})$ on TV 3D US & $8(23.5 \%)$ & $23(29.1 \%)$ & $31(27.4 \%)$ \\
Subgroup 2, IILFM $(1-4.9 \mathrm{~mm})$ on TV 3D US & $7(20.6 \%)$ & $12(15.2 \%)$ & $19(16.8 \%)$ \\
Subgroup 3, IILFM $(5-9.9 \mathrm{~mm})$ on TV 3D US* & $7(20.6 \%)$ & $35(44.3 \%)$ & $42(37.2 \%)$ \\
Subgroup 4, IILFM $(\geq 10 \mathrm{~mm})$ on TV 3D US* & $12(35.8 \%)$ & $9(11.4 \%)$ & $21(18.6 \%)$ \\
\hline
\end{tabular}




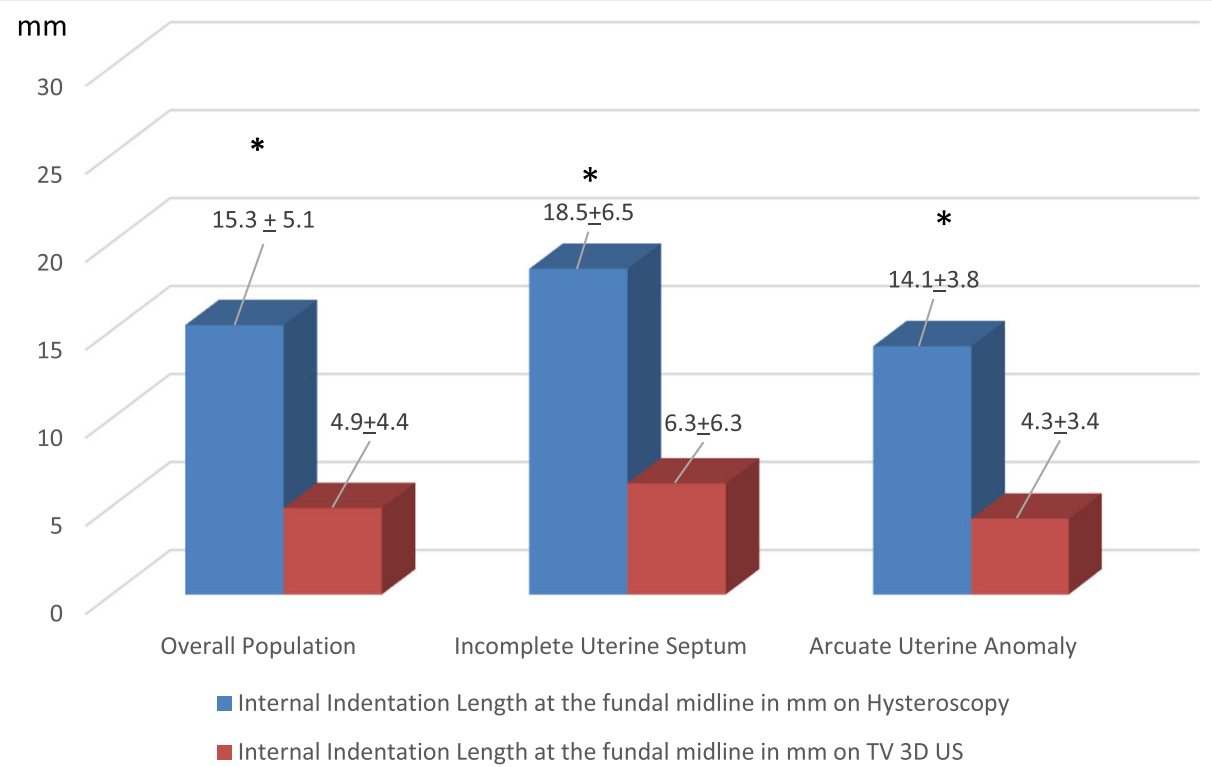

Fig. 5 Comparison between the mean IILFM measured in millimeters on hysteroscopy and on TV 3D US in patients with PSUAA, with PSUOA, and in the overall population

intrauterine scar tissue, uterine septum, PSUAA, and PSUOA. It also allows one to plan the proper surgical procedures in patients with these pathologies. As an example, in patient with uterine septum undergoing hysteroscopic correction, it allows for measurement of the width of the septum and the thickness of the myometrium in the fundal region, which makes division of the septum a safer procedure. Therefore, in patients with RPL, we advocate the use of TV 3D US in screening for PSU, and we strongly suggest to proceed with a diagnostic hysteroscopy to confirm the diagnosis, even if IILFM appears to be insignificant or there is no evidence of any IILFM on TV 3D US.

The notion that some patients who are classified as having unexplained RPL may, in fact, have some subtle PSU, should be entertained based on the data presented in our study, and also in view of the fact that more than $50 \%$ of patients with RPL can subsequently have a live birth. It has been illustrated before that many patients with uterine anomalies, even those with a septate uterus, may still have live birth [21]. Such variable reproductive outcomes, miscarriage versus live birth, in these patients, depend on the location of the placenta in relation to the uterine septum $[22,23]$. Miscarriage is more likely to happen if the placenta is located on the uterine septum, while live birth can occur when the placenta is located on the anterior, posterior, or lateral uterine walls [22, 23].

This is the first study to suggest, that in patients with unexplained RPL, TV 3D US is not totally accurate in measuring IILFM of PSUAA or PSUOA, when compared to a diagnostic hysteroscopy. Measurement on TV 3D US consistently underestimated the IILFM compared to the actual measurement on diagnostic hysteroscopy. In turn, our data suggest that there may be an element of uterine factor in some patients with unexplained RPL that goes undiagnosed if one relies only on TV 3 D US. It is not clear as to why the measurement of IILFM of PSUAA and PSUOA on TV 3D US is not accurate. However, volume transvaginal ultrasound pictures are computer generated, and therefore, it is possible that the IILFM in the generated pictures is not accurate.

Published data in the literature suggest that TV 3D US is the gold standard for the diagnosis of PSU, with a high degree of sensitivity and specificity [10, 11, 24]. However, Moini et al. [25] compared the findings on TV 3D US to hysteroscopy and laparoscopy in patients with suspected uterine septum and reported that TV 3D US was more accurate in septate uterus and less accurate in PSU [25]. The latter findings are similar to those in our study. Few studies suggested that TV 3D US is accurate in diagnosis of PSU based on comparison with the findings on office hysteroscopy [10, 26, 27]. However, other investigators suggested that the reproducibility of diagnosing intrauterine abnormalities through office hysteroscopy appeared to be disappointing [28-30]. In addition, a recent video abstract suggested that even a diagnostic hysteroscopy, performed under modified general anesthesia, can miss the diagnosis of such anomalies if uterine distension is suboptimal [31]. The latter commonly happens during office hysteroscopy to avoid patients' discomfort [29, 30, 32].

It is important to discuss our findings in context with ESHRE/ESGE classification [20]. This new classification has been criticized for leading to over diagnosis that may lead to over treatment [33]. A recent publication 
suggested the ESHRE-ESGE cutoff value overestimates the prevalence of PSU, while that of ASRM underestimates its prevalence, leaving in the gray zone most of the uteri that experts considered as partial septate [33, 34]. The authors of this report recommended considering indentation depth $\geq 10 \mathrm{~mm}$ as PSU, and this criterion is in agreement with expert opinion [33]. This is the IILFM that we used to define the presence of PSU on hysteroscopy in our study [33]. We would like to stress that the IILFM that we utilized $(\geq 10 \mathrm{~mm})$ was an arbitrary cutoff value that we chose, which happened to be in agreement with expert opinion [33].

In a prospective study of patients with recurrent pregnancy loss, who were suspected to have septate, and PSU, TV 3D US was extremely accurate in making the diagnosis of such anomalies, as confirmed on subsequent diagnostic hysteroscopy and laparoscopy [10]. In the same study, the authors reported that a negative study on TV 3D US was also accurate in ruling out such anomalies as confirmed on subsequent office hysteroscopy [10]. In contrast, $27.4 \%$ of the patients in our study were found to have no evidence of any internal indentation on TV 3D US $(0.00 \mathrm{~mm})$ [subgroup1]. Therefore, our findings are not in agreement with those of Ghi et al. [10]. If the recommendation by Ghi et al. [10] is followed, $27.4 \%$ of the patients in our study (subgroup 1) would have been considered to have no PSU based on TV 3D US [10]. In addition, another $16.8 \%$ of the patients in our study with IILFM of $1-4.9 \mathrm{~mm}$ (subgroup 2) would have also been considered normal with respect to such uterine anomaly based on TV 3D US. Furthermore, an additional $37.2 \%$ of the patients in our study with IILFM of 5-9.9 mm (subgroup 3) would also have been considered a variant of normal based on TV 3D US. This group of patients (subgroup 3) with IILFM of 5-9.9 mm on TV 3D US would have been considered to have insignificant IILFM, irrespective of the appearance of the central point of indentation (acute angle $<90^{\circ}$ as in PSUAA or obtuse angle $>90^{\circ}$ as in PSUOA) according to recent ASRM guidelines [34]. In such patients, a hysteroscopy would not be recommended and, in turn, the diagnosis would have been missed. It is worth noting that even if the new classification of ESHRE/ESGE regarding uterine septum (IILFM $\geq 50 \%$ of myometrial thickness), without our modification of the criteria (IILFM $>10 \mathrm{~mm}$ ), was used in our study, only $55.8 \%$ of the patients (subgroup 3 and subgroup 4) would have been suspected to have PSU [20].

Our study has its limitations. First, it is a retrospective study with all the limitations related to this design. Another limitation is the fact that the technique used to measure IILFM has not been validated, and it may not be perfectly accurate. However, to our knowledge, the technique described in this manuscript is the first attempt at measuring IILFM during diagnostic and operative hysteroscopy using a straight loop electrode. Therefore, prospective studies by other investigators are needed to validate this technique of measurement of IILFM. In future studies, the use of a novel graduated intrauterine palpator described recently by Di Spiezio Sardo et al. [35] can enhance the accuracy of measurement of IILFM on hysteroscopy [35]. The strength of this study stems from the fact that all the data is from one center, which eliminates variability in diagnostic measures by the operators.

\section{Conclusion}

Data in this study suggest that in patients with unexplained RPL, who were found to have PSU on hysteroscopy, IILFM can be underestimated on TV 3D US. We suggest that such patient should undergo further evaluation of the endometrial cavity with a diagnostic hysteroscopy to determine if they have PSU. Our findings may have tremendous implications in management of patients with unexplained RPL. Additional prospective studies are needed to confirm our findings. 9

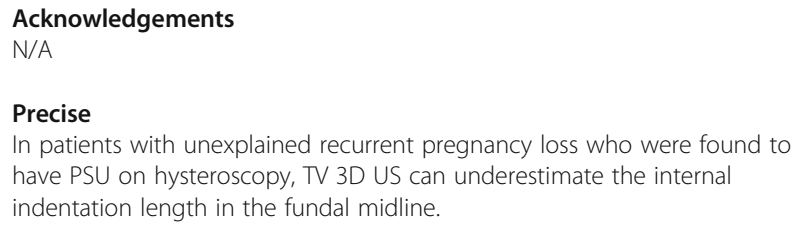

\section{Authors' contributions}

OA contributed to the protocol development, data collection, data analysis, and manuscript writing. JIC contributed to the protocol development, data analysis, and manuscript editing. JH contributed to the manuscript editing. MIA contributed to the protocol development, provision of study patients, data collection, data analysis, and manuscript editing. RW contributed to the manuscript editing. All authors read and approved the final manuscript.

\section{Funding}

The study did not receive external funding.

Availability of data and materials

Data can be available upon request.

Ethics approval and consent to participate

This retrospective study received an exemption from the Institutional Review Board at Hurley Medical Center, Flint, Michigan.

\section{Consent for publication}

All the authors consent for publication of this manuscript.

Competing interests

The authors declare that they have no competing interests.

\section{Author details}

1'Department of Obstetrics and Gynecology, Hurley Medical Center and Michigan State University College of Human Medicine, Flint Campus, Flint, Michigan, USA. ${ }^{2}$ Department of Research, Hurley Medical Center and Michigan State University College of Human Medicine, Flint Campus, Flint, Michigan, USA. ${ }^{3}$ IVF Michigan Rochester Hills and Flint PC, Rochester Hills, Michigan, USA. ${ }^{4}$ Division of Reproductive Endocrinology and Infertility, Department of Obstetrics and Gynecology, Hurley Medical Center and Michigan State University College of Human Medicine, Flint Campus, Flint, MI, USA. ${ }^{5}$ Division of Maternal Fetal Medicine, Department of Obstetrics and Gynecology, Hurley Medical Center and Michigan State University College of Human Medicine, Flint Campus, Flint, MI, USA. 
Received: 12 July 2019 Accepted: 5 November 2019

Published online: 11 December 2019

\section{References}

1. Stephenson M (1996) Frequency of factors associated with habitual abortion in 197 couples. Fertil Steril. 66:24-29

2. Branch DW, Gibson M, Silver RM (2010) Clinical practice: recurrent miscarriage. N Engl J Med. 363:1740-1747

3. Stephenson M, Kutteh W (2007) Evaluation and management of recurrent early pregnancy loss. Clin Obstet Gynecol. 50:132-145

4. Murugappan G, Shahine LK, Perfetto CO, Hickok LR (2016) Lathi RB (2016). Intent to treat analysis of in vitro fertilization and preimplantation genetic screening versus expectant management in patients with recurrent pregnancy loss. Hum Reprod. 31:1668-1674

5. Sugiura-Ogasawara M, Ozaki Y, Katano K, Suzumori N, Kitaori T, Mizutani E (2012) Abnormal embryonic karyotype is the most frequent cause of recurrent miscarriage. Hum Reprod. 27:2297-2303

6. Hodes-Wertz B, Grifo J, Ghadir S, Kaplan B, Laskin CA, Glassner M, Munne S (2012) Idiopathic recurrent miscarriage is caused mostly by aneuploid embryos. Fertil Steril. 98(3):675-580

7. Chan Y, Jayaprakasan K, Zamora J, Thornton JG, Raine-Fenning N, Coomarasamy A (2011) The prevalence of congenital uterine anomalies in unselected and high-risk populations: a systematic review. Hum Reprod Update. 17(6):761-771

8. Grimbizis G, Camus M, Tarlatzis BC, et. al. Clinical implications of uterine malformation and hysteroscopic treatment results. Hum Reprod Update. 2001;7(2):161-174.

9. Homer HA, Li TC, Cooke ID (2000) The septate uterus: a review of management and reproductive outcome. Fertil Steril. 73(1):1-14

10. Ghi T, Casadio P, Kuleva M, et. al. Accuracy of three-dimensional ultrasound in diagnosis and classification of congenital uterine anomalies. Fertil Steril. 2009;92(2):808-813

11. Graupera B, Pascual MA, Hereter L et al (2005) Accuracy of threedimensional ultrasound compared with magnetic resonance imaging in diagnosis of Mullerian duct anomalies using ESHRE-ESGE consensus on the classification of congenital anomalies of the female genital tract. Ultrasound Obstet Gynecol. 46(5):616-622

12. Kallia N, Abuzeid O, Ashraf M, Abuzeid M. Role of hysteroscopy in diagnosis of subtle uterine anomalies in patients with normal hysterosalpingography. Fertil Steril. 2011;96(3) Supplement, pp S12, 0-40.

13. Abuzeid O, Abdullah A, Moustafa ASZ, Hebert J, Rocha F, Abuzeid MI. Limitations of radiological screening tests in detection of subtle incomplete septum or arcuate uterine anomaly in patients with recurrent pregnancy loss (RPL). Hum Reprod. 2016:Supplement, 23(7):S187.

14. Abuzeid M, Abuzeid O. Three-dimensional ultrasonography of subtle uterine anomalies: correlation with hysterosalpingogram, two-dimensional ultrasonography, and hysteroscopy. In: Ultrasonography in gynecology. Editors: Botros Rizk and Elizabeth Puscheck. Cambridge University Press 2014, Chapter 8, pp 66-79.

15. Abuzeid O, Zaghmout O, Corrado J, Hebert J, Ashraf M, Abuzeid M. Comparison between the findings on trans-vaginal 3D ultrasound scan and hysteroscopy in patients diagnosed with subtle incomplete uterine septum/ arcuate uterine anomaly on hysteroscopy. Fertil Steril. 2015;104(3), Supplement, pp e28, 0-70.

16. Abuzeid O, Zaghmout O, Corrado J, Hebert J, Abuzeid M (2016) Three-dimensional ultrasonography with or without saline infusion sonogram in detecting subtle uterine anomalies: correlation with hysteroscopy. JMIG. 23(7):S145

17. Abuzeid M, Ghourab G, Abuzeid O, Mitwally M, Ashraf M, Diamond M (2014) Reproductive outcome after IVF following hysteroscopic division of incomplete uterine septum/arcuate uterine anomaly in women with primary infertility. Facts Views Vis ObGyn. 6(4):194-202

18. (1988) The American Fertility Society classifications of adnexal adhesions, distal tubal occlusion, tubal occlusion secondary to tubal ligation, tubal pregnancies, Müllerian anomalies and intrauterine adhesions. Fertil Steril 49:944-955

19. Mitwally MFM, Abuzeid M. Operative hysteroscopy for uterine septum. In: Infertility and Assisted Reproduction. Editors: Botros R. M. Rizk, Juan A. GarciaVelasco, Hassan N. Sallam and Antonis Nakrigiannakis. Cambridge University Press 2008, Part II Infertility Evaluation and Treatment, Chapter 13,115-131.

20. Grimbizis GF, Gordts S, Di Spiezio SA et al (2013) The ESHRE/ESGE consensus on the classification of female genital tract congenital anomalies. Hum Reprod. 28(8):2032-2044
21. Heinonen P (2006) Reproductive surgery: complete septate uterus with longitudinal vaginal septum. Fertil Steril. 85(3):700-705

22. Fedele L, Dorta M, Brioschi D, Guidici MN, Candiani GB (1989) Pregnancies in septate uteri: outcome in relation to site of uterine implantation as determined by sonography. AJR. 152:781-784

23. Abuzeid O, Zaghmout O, Herbert J, Rocha F, Abuzeid M (2016) Placental location and reproductive outcome in patients with uterine septum or arcuate uterus. Ob \& Gyn 127:64S-19G

24. Jurkovic D, Geipel A, Gruboeck K (1995) Three-dimensional ultrasound for the assessment of uterine anatomy and detection of congenital anomalies: a comparison with hysterosalpingography and two-dimensional sonography. Ultrasound Obstet Gynecol. 5(4):233-237

25. Moini A, Mohammadi S, Hosseini R, Eslami B, Ahmadi F (2013) Accuracy of 3-dimensional sonography for diagnosis and classification of congenital uterine anomalies. J Ultrasound Med. 32:923-927

26. Faivre E, Fernandez H, Deffieux X, Gervaise A, Frydman R, Levaillant JM (2012) Accuracy of three-dimensional ultrasonography in differentia diagnosis of septate and bicornuate uterus compared with office hysteroscopy and pelvic magnetic resonance imaging. JMIG. 19(1):101-106

27. Lagana AS, Ciancimino L, Mancuso A, Chiofalo B, Rizzo P, Triolo O (2014) 3D sonohysterography vs hysteroscopy: a cross-sectional study for the evaluation of endouterine diseases. Arch Gynecol Obstet. 290:1173-1178

28. Kasius JC, Broekmans FJM, Veersema S, et. al. Observer agreement in the evaluation of the uterine cavity by hysteroscopy prior to in vitro fertilization. Hum Reprod. 2011;26(4):801-807.

29. Smit JG, Kasius JC, Eijkemans MJC et. al. The international agreement study on the diagnosis of the septate uterus at office hysteroscopy in infertile patients. Fertil Steril. 2013;99(7):2108-2113.

30. Smit JG, Overdijkink S, Mol BW et. al. The impact of diagnostic criteria on the reproducibility of the hysteroscopic diagnosis of the septate uterus: a randomized controlled trial. Hum Reprod. 2015:30(6):1323-1330.

31. Abuzeid O, Raju R, Hebert J, Abuzeid M (2016) Effect of proper uterine distention on the detection rate of subtle uterine anomalies during hysteroscopy. JMIG. 23(7):S134-S135

32. Ludwin A, Ludwin I, Kudla M, et. al. Diagnostic accuracy of threedimensional sonohysterography compared with office hysteroscopy and its interrater/intrarater agreement in uterine cavity assessment after hysteroscopic metroplasty. Fertil Steril. 2014;101(5):1392-1399.

33. Ludwin A, Martins WP, Nastri CO et. al. Congenital uterine malformation by experts (CUME): better criteria for distinguishing between normal/arcuate and septate uterus. Ultrasound Obstet Gynecol. 2018;51(1):101-109.

34. The American Society for Reproductive Medicine. Uterine septum: a guideline (2016) Practice Committee of the American Society for Reproductive Medicine. Fertil Steril. 106(3):530-540

35. Di Spiezio SA, Zizolfi B, Bettocchi S et al (2016) Accuracy of hysteroscopic metroplasty with the combination of presurgical 3-dimensional ultrasonography and a novel graduated intrauterine palpator: a randomized controlled trial. JMIG. 23(4):557-566

\section{Publisher's Note}

Springer Nature remains neutral with regard to jurisdictional claims in published maps and institutional affiliations.

\section{Submit your manuscript to a SpringerOpen ${ }^{\odot}$ journal and benefit from:}

- Convenient online submission

- Rigorous peer review

- Open access: articles freely available online

- High visibility within the field

Retaining the copyright to your article

Submit your next manuscript at $>$ springeropen.com 